\title{
"It's in my other hand!" - Studying the Interplay of Interaction Techniques and Multi-Tablet Activities
}

\author{
Johannes Zagermann, Ulrike Pfeil, Philipp von Bauer, Daniel Fink, and Harald Reiterer \\ HCI Group, University of Konstanz \\ \{johannes.zagermann,ulrike.pfeil,philipp.bauer,daniel.immanuel.fink,harald.reiterer\}@ uni.kn
}

\begin{abstract}
Cross-device interaction with tablets is a popular topic in HCI research. Recent work has shown the benefits of including multiple devices into users' workflows while various interaction techniques allow transferring content across devices. However, users are only reluctantly using multiple devices in combination. At the same time, research on cross-device interaction struggles to find a frame of reference to compare techniques or systems. In this paper, we try to address these challenges by studying the interplay of interaction techniques, device utilization, and task-specific activities in a user study with 24 participants from different but complementary angles of evaluation using an abstract task, a sensemaking task, and three interaction techniques. We found that different interaction techniques have a lower influence than expected, that work behaviors and device utilization depend on the task at hand, and that participants value specific aspects of crossdevice interaction.
\end{abstract}

\section{Author Keywords}

cross-device interaction; interaction techniques; evaluation.

\section{CCS Concepts}

-Human-centered computing $\rightarrow$ Human computer interaction (HCI); User studies;

\section{INTRODUCTION}

Research on cross-device interaction is based on the idea that users easily interact with multiple devices, not recognizing the connected devices as single entities but perceiving them as components of the same underlying system, reflecting Weiser's vision for the computer for the 21st century [45]. The analyses of user behavior and strategies in cross-device systems suggest that while participants own several devices [7], creatively combine them [33], and are able to cope with the simultaneous use of multiple devices (e.g., [11, 12, 44]), they are hesitant to fully exploit the potential benefits of a large ecology of devices due to a legacy bias of being used to working with single devices only $[14,35]$. Findings suggest that performance, device utilization, and work strategy heavily depend on the task at hand

Permission to make digital or hard copies of part or all of this work for personal or classroom use is granted without fee provided that copies are not made or distributed for profit or commercial advantage and that copies bear this notice and the full citation on the first page. Copyrights for third-party components of this work must be honored For all other uses, contact the Owner/Author(s).

CHI '20, April 25-30, 2020, Honolulu, HI, USA.

(C) 2020 Copyright is held by the owner/author(s)

ACM ISBN 978-1-4503-6708-0/20/04.

http://dx.doi.org/10.1145/3313831.3376540

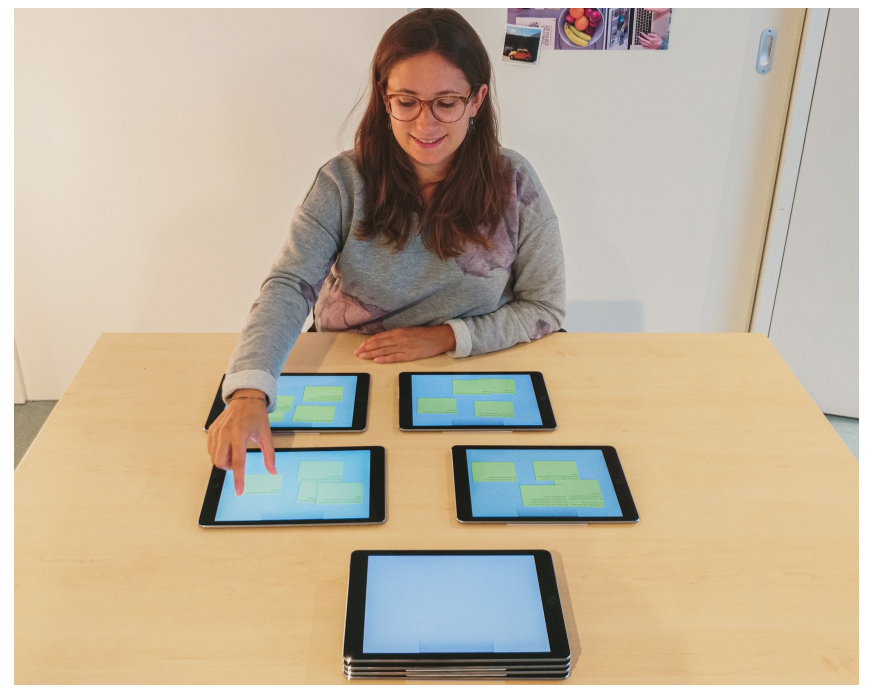

Figure 1. A user demonstrates possible cross-device interactions in our multi-tablet environment.

with a tendency for users to under-utilize provided devices. In order to address these issues, existing research has designed and investigated techniques for connecting devices (e.g., [20, $37,40]$ ), integrating devices into workflows (e.g., [2, 21, 24, $30]$ ), and transferring content across devices (e.g., [27, 34, 38]). Findings from these studies show that interaction techniques impact task performance, which raises the question of their potential to alleviate the burden of device utilization across different domain-specific tasks [14].

Consequently, previous research on cross-device interaction has shown various strategies to evaluate systems and techniques that connect and combine multiple devices [4]. While different analytical and empirical approaches for various stages during the design process exist, the overall evaluation methodology for cross-device work is still struggling to find a frame of reference to compare or evaluate existing and future techniques and systems, resulting in a rather fragmented research landscape. Brudy et al. [4] highlight the need for improving evaluation strategies to finally "unify empirical and technical cross-device work into one stream of research."

Our work builds on existing cross-device research and aims to shed light on the interplay of interaction techniques, device utilization, and task-specific work behavior. Although many techniques for cross-device interaction exist and have been studied extensively, the difficulties of successfully interweaving the techniques with the setting (e.g., tasks and devices) and 
ensuring viable cross-device interaction remain [14, 25, 29]. We tackle this challenge with a user study that provides different but complementary angles of evaluation using an abstract classification task and a less restricted sensemaking task. This methodological combination results in a holistic evaluation approach with different types of tasks and both, qualitative and quantitative measurements that provided insights in the comparison of interaction techniques, their impact on device utilization, and their adoption across tasks. We identified relevant patterns, which lead to the following findings:

Finding 1: Regardless of the task, the influence of interaction techniques on participants' activities is lower than expected. Task completion times slightly differ, and participants prefer unfamiliar yet faster techniques.

Finding 2: Regardless of the interaction technique, participants develop different problem-solving strategies, facilitated by high utilization of devices and different quality features of cross-device interaction.

Finding 3: An extensive training in using a large ecology of devices in an artificial task can lead to a high device utilization in a subsequent sensemaking task.

This paper successfully takes on the challenge to investigate the interplay of interaction techniques, device utilization, and task-specific work behavior in cross-device settings. Besides the individual findings of the user study, the key contribution of our paper is the methodological approach of combining different but complementary angles of evaluation that allowed us to dig deeper into the subtleties of cross-device interaction.

\section{RELATED WORK}

From a developer perspective, some frameworks allow connecting devices and thus support interaction across devices (e.g., $[13,26,36])$. Also, there is an area of research that investigates how multiple devices can be integrated into workflows (e.g., [2, 21, 24, 30]) and Brudy et al. [4] provide a comprehensive overview of research on cross-device interaction in general. While these areas provide insights into how cross-device interaction can be facilitated, we review work on tablet-focused systems to inform the apparatus of our study. Also, we summarize studies on cross-device interaction techniques for object transfer across devices to inform the choice of our investigated interaction techniques and describe evaluation strategies for cross-device interactions.

\section{Cross-Device Systems}

Recent research has investigated the influence of a shared tabletop [44] and its size [48] on collaborative activities using sensemaking tasks. Results show that users can efficiently collaborate using tablet-size devices [48]. Brudy et al. [3] continued to investigate the role of a shared tablet-size device in collaborative activities and showed that an additional overview device can extend periods of closely-coupled collaboration. Wozniak et al. [46] presented a system that allows for collaborative activities with spatially-aware tablets - and compared it to a paper-based and tabletop condition using a sensemaking task. Their system revealed faster task completion times compared to paper and similar results for task load compared to the tabletop condition. Hamilton and Wigdor [9] have shown that participants can quickly adapt to unfamiliar settings and make use of up to ten mobile devices in a tablet-only setting working on a sensemaking task. Chen et al. [5, 6] investigated active reading activities using a multi-tablet system. Compared to paper, their system provides enhanced navigation within documents and allows for synchronization across multiple devices. Plank et al. [35] built on this research and investigated the usage of mobile devices in a tablet-only setting for collaborative visual analytics. In contrast to previous work [9], Plank et al.'s participants did not exhaust all available devices and solved the tasks with one or two devices. The authors refer to a legacy bias that influences participants how they deal with mobile devices, resulting in the usage of fewer tablets than expected.

This related work on cross-device systems shows the versatility of mobile devices and inspired us to use tablets for our user study. The described studies analyzed the use of tablets for different activities (e.g., sensemaking or visual analytics tasks), leading partly to contrasting results regarding tablet utilization. We believe that this might not only be linked to a legacy bias [35], but also to the task at hand. This inspired us to control the number of devices-to-be-used in our abstract classification task and to investigate if the training to use such a large ecology of devices impacts device utilization in a subsequent sensemaking task as proposed by [35].

\section{Cross-Device Interaction Techniques}

In addition to device utilization, we were interested in the impact of interaction techniques on users' work behavior for different tasks. In the following, we review related work that informed the choice of our investigated interaction techniques.

Rädle et al. [38] investigated if user interfaces for transferring documents across devices should provide spatial information about nearby devices. They compared a menu-based approach (similar to [9]), without information on the location of nearby devices to two spatially-aware techniques using their HuddleLamp [37]. Results show that spatially-aware techniques (e.g., dragging an item 'through' a portal-like area to another device) were preferred and lowered participants' task load, yet the menu-based approach was beneficial for settings where the spatial configuration of devices is not changing rapidly. As the menu-based approach proved to be a sound state-of-the art baseline [38], we decided to include a menu-based interaction technique (MENU) in our study.

With portal-like techniques (e.g., [27, 38]), content can be transferred to a nearby device by dragging it 'through' a portal over the device's edge. Building on this research, Jokela et al. [19] compared a technique called Tray to two techniques that rely on physical contact by participants with devices (Transfer Mode) and devices themselves (Device Touch). Participants liked the visual feedback and familiar handling of Tray, performed faster with the Transfer Mode, and considered Device Touch as rather cumbersome. Similar to Tray and Portal, Scott et al. [41] compared their Bridge and a Pick and Drop technique for object transfer between a tabletop and mobile devices. Both techniques effectively supported participants in their tasks. Bridge resulted in a lower cognitive effort, whereas Pick and Drop was seen as more efficient as participants could 
pick multiple items sequentially and drop them all at once. In line with these studies, we chose to include a portal-like interaction technique called TRAY in our study.

Rekimoto originally described Pick and Drop [39]: The technique allows to pick up an object with a pen on one display and release it at another device by touching the display - creating the illusion of the user manipulating a physical object. Paay et al. [34] compared their variant of the Pick and Drop technique called Pinch with three other techniques called Swipe, Swing, and Flick for interactions between a mobile device and a large display using additional mid-air gestures, taking a first step to find relative strengths and weaknesses of the named interaction techniques. We built on this previous research comparing existing interaction techniques and included a gesture-based condition called PICK'n'DROP in our study.

This work on cross-device interaction techniques shows a variety of ways to transfer information across devices. Scenarios include mobile devices only (e.g., $[19,38]$ ), tablets and tabletops [41], and mobile devices and wall-displays [34]. Tasks range from pre-defined workflows [38], to image sorting [19], and games [41]. This inspired us to investigate not only relative strengths and weaknesses of existing interaction techniques but also their interdependence with task-specific work behavior and device utilization.

\section{Evaluating Cross-Device Interactions}

Brudy et al. offer a comprehensive overview of evaluation strategies for cross-device interactions in their cross-device taxonomy [4]. The authors distinguish between evaluation through a) demonstration or b) usage, c) heuristic evaluation, d) informative studies, and e) technical evaluation. While the first two strategies allow investigating how users apply a novel technique and how usable it is; heuristic and technical evaluations assess the qualities of a system or technique from an analytical point of view. Informative studies, however, provide "insights into users' needs" [4], and help to understand the usage of cross-device systems to guide future research. Brudy et al. [4] emphasize that more work is necessary for improving evaluation strategies for cross-device interactions and that current strategies are disconnected from each other. The authors point out that finding a frame of reference that allows evaluating techniques and systems is one of the key challenges for future research on cross-device interactions.

This separation of different evaluation strategies inspired us to look beyond the boundaries of a single strategy and combine different but complementary angles of evaluation. We did this with a methodological combination of two evaluation strategies: An abstract classification task allowed us to study aspects of usability and a less-restricted sensemaking task helped us to build an understanding of how users work with a large ecology of devices.

\section{RESEARCH QUESTIONS}

The goal of our study was to better understand the implications of different cross-device interaction techniques and to contribute a nuanced description of their effects and influences on users' working behaviors. We address this overall research question by investigating the following subquestions both qualitatively and quantitatively:

$\boldsymbol{R Q 1}$ - How do cross-device interaction techniques influence work performance? (measured in different aspects of performance and subjective user rankings)

$\boldsymbol{R Q 2}$ - How do cross-device interaction techniques influence work behaviors? (video analysis to find work behaviors)

$\boldsymbol{R Q 3}$ - How are cross-device interaction techniques, device utilization, and task-specific activities interrelated? (measured in user descriptions and analysis of data $\operatorname{logs}$ )

A distinctive attribute of our study is that we tackle these questions not only using qualitative and quantitative metrics but also from different perspectives: 1) Using an abstract task (see Task 1 -Abstract Classification Task) allows us to investigate $\boldsymbol{R Q 1}$ and $\boldsymbol{R Q 2}$ to guarantee for a high internal validity. 2) Providing the ability to choose which technique to use in combination with a sensemaking task (see Task 2 - The Murder Mystery) provides a less restricted and more realistic scenario that allows to study participants' activities with higher ecological validity (RQ3). Additionally, this allows to see how participants include their preferred technique a) in general or for specific sub-tasks, b) in combination with other techniques, and c) to explore how interaction techniques, utilization of devices and task-specific activities are interrelated. This methodological combination results in a holistic evaluation approach that does not only provide qualitative and quantitative insights into relative strengths and weaknesses of interaction techniques (similar to [11]) and the usage of a large ecology of devices but can also serve as a first step of an evaluation framework for future cross-device interaction [4].

\section{USER STUDY}

We conducted a lab study with the two within-subjects factors task and interaction technique. The order of task was predefined, with all participants finishing task 1 before starting task 2. We counterbalanced the factor interaction technique with the order of the techniques only for task 1 and allowed participants to use the techniques as favored in task 2 (similar to [11]). This study design allowed us to compare the techniques independently (task 1) and explore how participants use the techniques and utilize the provided large ecology of devices (task 2). The remainder of this section provides an overview of the interaction techniques, the system in general, the two tasks, participants, procedure, apparatus, and data collection.

\section{Interaction Techniques}

Concerning the interaction techniques, we defined three conditions: 1) MENU, 2) TRAY, and 3) PICK'n'DROP. They constitute three types of state-of-the-art and robust-to-use interaction techniques (see Related Work). These techniques do not require a) additional user engagement with devices (e.g., rotating or tilting) or b) extra hardware augmentation (e.g., tracking cameras), which allows for a valid comparison of techniques and an investigation of technique-independent device utilization across both tasks. We decided to allow participants to transfer multiple sticky notes at the same time as Scott et al. [41] have shown that this increases efficiency. 


\section{Interaction Technique 1 - MENU}

The first interaction technique was inspired by interactions based on context-menus: Pop-up and context-menus are standard features of every day WIMP-applications (e.g., copying and pasting text from one application to another), mobile devices (e.g., copying and pasting locations from map apps to messaging apps), or across different types of devices (e.g., using Apple's Universal Clipboard ${ }^{1}$ ). Similarly, we designed MENU as follows (see Figure 2): The MENU appears by performing a long press interaction $(200 \mathrm{~ms})$ on a sticky note. Tapping on the now visible cut-button cuts this sticky note. Now, users can perform another long-press interaction on any position on any tablet - triggering the paste-button to appear. Tapping on it will paste the sticky note at this position. To cut multiple sticky notes, the MENU has to be used sequentially. Multiple pasted sticky notes are piled with a randomized offset of a few pixels to increase visibility.

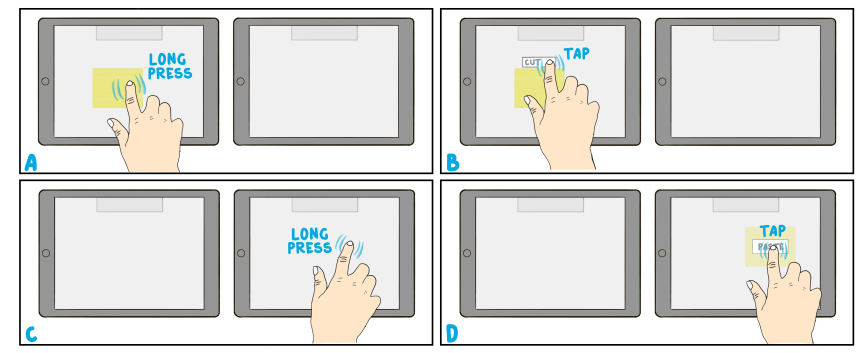

Figure 2. Transferring a sticky note using MENU.

\section{Interaction Technique 2 - TRAY}

The second interaction technique was inspired by previous research (e.g., [27, 41, 42]) that has shown that participants can benefit from drag and drop interactions, where the drop target creates a digital bridge between devices. We designed TRAY as follows (see Figure 3): A digital tray was shown in the middle of and at the upper edge of each tablet. Sticky notes could be transferred by dragging and dropping them on the TRAY. As a result, the size of the sticky note was decreased to fit the TRAY - maintaining the readability of the sticky note's text - and the sticky note was then visible on every tablet in the area of the TRAY.

By dragging the sticky note out of the TRAY and dropping it on any surface, it is transferred to the currently used tablet. With this technique, users are also able to transfer one or multiple sticky notes at the same time across multiple devices.

\section{Interaction Technique 3-PICK'n'DROP}

The third interaction technique was inspired by Rekimoto's same-named technique [39]. In contrast to Rekimoto and in line with recent research $[15,34]$, we allow users to pick and drop sticky notes with their fingers. In our case, PICK'n'DROP makes use of the well-known pinch-to-zoom technique (see Figure 4). Instead of changing their size while performing a pinch gesture, sticky notes are picked up (i.e., cut) when a pinch-in gesture is performed on them. Performing a pinch-out gesture on, e.g., another tablet, drops this

\footnotetext{
${ }^{1}$ Apple Universal Clipboard: https: //support. apple.com/en-us/ HT209460, last accessed: 2020-1-8
}

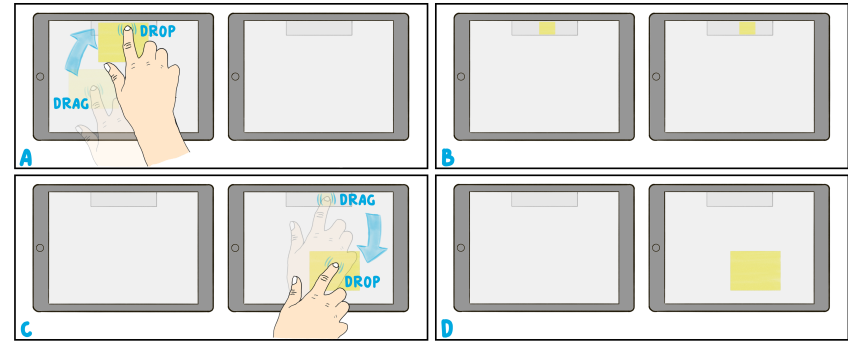

Figure 3. Transferring a sticky note using TRAY.

sticky note at that position. With this technique, users are also able to transfer one or multiple sticky notes at the same time across devices by picking them sequentially and dropping them. Multiple pasted sticky notes are piled with a randomized offset of a few pixels to increase visibility.

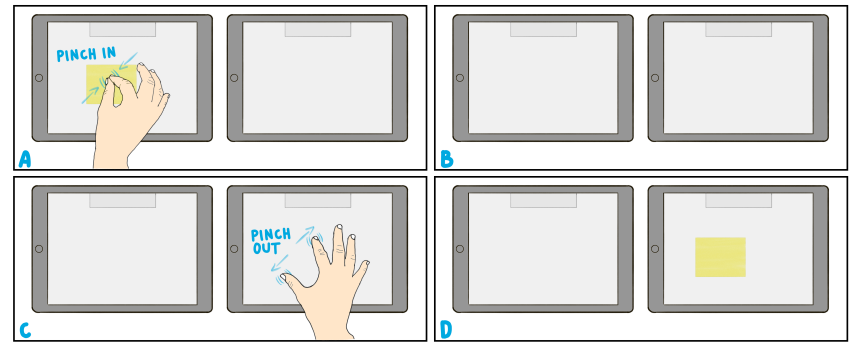

Figure 4. Transferring a sticky note using PICK'n'DROP.

\section{System Description}

Our system enables users to use multiple tablets to work with pieces of information - represented as digital sticky notes on two levels: Users can a) freely move and arrange sticky notes on a single tablet and b) transfer them across multiple tablets using the described interaction techniques. Especially for the second task (see Task 2 - The Murder Mystery), this allows for spatial arrangements to prioritize and compare information [44] and to find connections between different events, persons, and places. We implemented a web-based system using the JavaScript framework Meteor without additional hardware augmentation (e.g., camera tracking systems) that allows for ad hoc cross-device use [4].

\section{Tasks}

We used two different tasks for our study: In the first task, we chose to use an abstract classification task as it guaranteed a high internal validity when comparing different interaction techniques. We were also interested in the effects and influences of the different interaction techniques on participants working behavior using a more realistic sensemaking task.

\section{Task 1 - Abstract Classification Task}

To provide a documented and sufficiently demanding task, we were inspired by the abstract classification task used by Liu et al. [23], who studied the influence of display size on single users' interaction with a wall-sized display. The main components of the tasks are discs - labeled with different letters. Participants have to move these discs to their specific container (i.e., a display) in order to correctly classify them. The 
color of the discs helps participants to solve the task: If not correctly classified (i.e., in the wrong container), discs show a red background color and turn green when transferred into a display where all discs share the same label. The same task was used, e.g., by Jakobsen and Hornbæk [18] who investigated the influences of locomotion on single users' interaction with a wall-sized display. This task was also used to study collaborative activities, either co-located [22] or remote [1].

In light of the prevalent use of this task, we took it as inspiration for our first task. As the task itself was initially developed for wall-sized displays and mostly used for studies in such settings, we decided to adapt it to cross-device settings: Following previous research on the usage of cross-device systems (e.g., [9]) we decided to set the number of available tablets to 8 (see sections Related Work and Apparatus for detailed descriptions). Participants were allowed to have as many items on their tablets as they wished, yet we defined a total number of 40 items (represented using $8 \times 5$ letters), resulting in a final solution of 5 items per tablet in an ideal number of 35 item movements. This was a trade-off decision of having enough data without overwhelming the participants. Items are represented as sticky notes with a size that allows arrangement of 6 sticky notes per tablet with no overlap (in line with [23]). We decided to visualize items as sticky notes instead of discs to increase the comparability with the second task. Sticky notes could be moved across tablets using one of the interaction techniques. We labeled sticky notes with eight different letters: $\mathrm{C}, \mathrm{D}, \mathrm{E}, \mathrm{F}, \mathrm{H}, \mathrm{K}, \mathrm{N}$, and R. These letters were chosen as they guarantee equal legibility according to the BS 4274-1:2003 vision test standard [16]. Each letter was used to label five sticky notes with a standard computer font (font-size: 12pt).

We decided against a pre-configuration of correctly classified items (i.e., having 3 to 4 correctly classified items and only 1-2 wrong ones per tablet) as our pre-tests have shown that participants solved the task on different levels of difficulty in less than a minute. Thus, we decided to omit such a pre-configuration in favor of keeping the task sufficiently demanding. Also, a pre-configuration could influence workplace configurations. As we were interested in our participants' spatial configuration and utilization of the tablets, we decided to place all sticky notes initially on one tablet that was on top of the stack of the tablets. To ensure equal difficulty, we randomized the order in which sticky notes where represented initially.

\section{Task 2 - The Murder Mystery}

As a counterpart to our abstract classification task, we opted for a sensemaking task as a real-world activity to dig deeper into the subtleties of cross-device interaction. The "Stegosaurus" task and data set of the 2006 VAST challenge [8] was often used in previous publications, and recent research (e.g., [31, 48]) has shown that this task indeed offers insights into the usage of multiple devices, yet solving the task can be challenging and task completion times of up to 90 minutes or longer are no exceptions. Thus, we decided to opt for a less time-consuming but also complex sensemaking task: The murder mystery suggested by Stanford et al. [43] is a crime-solving task and was also used before by Wozniak et al. [46] in their multi-tablet system. In the task, multiple people were involved in a murder mystery, which happened during the night. Participants had to find the murderer, the weapon, the time and place of the murder, and the motive by combining clues and hints written on sticky notes (similar to [46]). As suggested [43, 46], we increased the difficulty by adding distracting information. In total, there were 40 pieces of information represented as sticky notes (font-size: $12 \mathrm{pt}$ ), which allows for better comparability between our two tasks. Participants had to understand the content, filter non-important information, and connect essential facts that lead to new insights and suppositions to come up with answers to the murder mystery.

\section{Participants}

24 participants (14 female, 10 male) were recruited for the study. The mean age was 23.88 years $(\mathrm{SD}=4.00)$. All participants had normal or corrected to normal eyesight; consequently, they had no problems with the size of the sticky notes or the used font size. 20 participants were undergraduate students - the other four participants were university employees or Ph.D. students. Participants had a mixed background ranging from psychology to biology, educational science, and law. All participants were familiar with touch interfaces and reported the frequent handling of mobile devices like tablets and smartphones. To recruit participants, we used postings and flyers looking for people who are interested in new technologies and crime mysteries at our local campus. This helped us to find participants who were motivated by our chosen tasks.

\section{Procedure}

At the beginning of the study, each participant was asked to fill out a questionnaire about demographics and tech-savviness. Then, each participant received an introduction into the first task. After that, the first interaction technique was shown using a demo item set. Participants had time to explore the functions of the system and the specific interaction technique with a demo item set until they felt comfortable using it. After that, the experimenter asked participants to start the first task with the first interaction technique with the remark to be as quick and accurate (e.g., as little interaction steps) as possible. After that, participants were asked to fill in a NASA TLX questionnaire [10]. This sequence was the same for all three interaction techniques. To avoid learning effects, we counterbalanced the order of interaction techniques and randomized the sequence of the shown sticky notes. After that, participants were asked to fill in a questionnaire concerning subjective preferences. Then, the experimenter introduced participants to the second task: They were asked to solve the murder mystery and were free to use any of the three different interaction techniques (cf., [11]) and as many tablets as desired. Finally, each participant filled out a post-questionnaire concerning clarification of the usage of the interaction techniques in this phase. Each session lasted about one hour in total, and we reimbursed participants for their time.

\section{Apparatus}

The setting consisted of an office table $(1.20 \times 0.80 \mathrm{~m})$ on which we stacked eight Apple iPads (9.7") in the middle of and at a long side of the table (cf. Figure 1). We attached felt pads to the bottom of each tablet for smoother movements (cf. [35]). Participants sat on a chair on the opposite 
side of the table. Next to them was a small additional desk for filling out questionnaires. We used a camera in bird's-eye view to see interactions, working behaviors, and device utilizations.

\section{DATA ANALYSIS}

We employed several data collection methods in order to investigate our research questions:

$\boldsymbol{R Q 1}$ - Performance: We measure task completion time, number of cross-device transfers, the ratio of object transfers per interaction, tablet utilization, and subjective rankings.

$\boldsymbol{R Q 2}$ - Work Behavior: To explore the work behavior, we analyzed participants' activities using video codings.

$R Q 3$ - Cross-Device Interrelations: We analyzed frequencies of cross-device interactions, utilization of tablets, and rationales of using the techniques for the murder mystery.

\section{Video Data}

We developed a coding scheme to analyze video data of the first task. We focused on participants' work behavior (e.g., sorting sticky notes). After the initial viewings of our videos, we started to distinguish between different codes that offer a sound description of the video data and apply to our research questions. This coding scheme was refined until we felt that it was saturated. An inter-coder reliability test was conducted for six samples (two per technique; in total, about 25 minutes) of video data to ensure objective coding by our two independent coders. Cohen's Kappa of 0.70 revealed substantial inter-coder reliability for the final coding scheme presented below.

$S$-Sorting: A participant was explicitly sorting sticky notes (e.g., piling all sticky notes with the letter "C") on one tablet.

D-Distributing: A participant distributed one or multiple sticky notes to another tablet.

$\boldsymbol{E}$ - Exploring: Similar to $S$ - Sorting, but in this case, participants were implicitly sorting sticky notes while explicitly searching for a sticky note in a pile of sticky notes.

We defined all codes as mutually exclusive state events. Thus, we were able to compare the duration of activities and their sequences. The video codes helped us to elicit the main aspects of work behavior. Also, it allowed us to focus on specific subparts of the video for detailed qualitative observations.

\section{Data Logs}

We collected logs of participants' interaction with the system, namely when and how often participants transferred sticky notes from one tablet to another (including an internal ID for each tablet), the ratio of object transfers per interaction, and task completion times. We did this to analyze whether the interaction technique affected how and how often participants interacted with sticky notes and tablets.

\section{Questionnaires}

We used a NASA TLX questionnaire [10] to investigate subjective task load caused by the three interaction techniques. In addition to this, we used one questionnaire for subjective preferences and another questionnaire after the final task to clarify participants' choice of used interaction techniques.

\section{FINDINGS}

We report our findings in relation to our research questions. We focus on differences across the three interaction techniques with respect to participants' performance $(\boldsymbol{R} Q 1)$, their work behavior (RQ2), and the interrelations of interaction techniques, device utilization, and task-specific activities (RQ3). As the data violated the assumption of a normal distribution, we decided to use a non-parametric approach to analyze the data statistically. For the overall tests on statistical significance, we used a Friedman test. If this test showed statistically significant differences, we employed a Wilcoxon signed-rank test as a post-hoc analysis, including Bonferroni correction.

\section{RQ1 - Performance | Abstract Classification Task}

We differentiate between task completion time, the number of sticky note transfers, the ratio of object transfers per interaction, tablet utilization, task load, and subjective preferences.

\section{Task Completion Time}

We measured the time participants needed to solve the task. Participants' mean times were $\mathrm{M}_{\mathrm{MENU}}=241.00 \mathrm{~s}(\mathrm{SD}=58.58)$, $\mathrm{M}_{\mathrm{TRAY}}=177.54 \mathrm{~s}(\mathrm{SD}=28.31)$, and $\mathrm{M}_{\mathrm{PICK}} \mathrm{n}^{\prime} \mathrm{DROP}=176.92 \mathrm{~s}$ $(\mathrm{SD}=36.27)$. This difference was statistically significantly different $\left(\chi^{2}(2)=25.00, p<.05\right)$. A post-hoc analysis revealed statistically significant differences between MENU and TRAY $(Z=3.972, p<.016)$ as well as between MENU and PICK'n'DROP $(Z=3.972, p<.016)$.

\section{Number of Transfers across Devices}

We measured how often participants transferred sticky notes across the available tablets. The optimal number of moves is 35. A Friedman test revealed no statistically significant differences when comparing the three different interaction techniques with $\mathrm{M}_{\mathrm{MENU}}=37.04(\mathrm{SD}=3.13), \mathrm{M}_{\mathrm{TRAY}}=36.13$, $(\mathrm{SD}=1.36)$, and $\mathrm{M}_{\mathrm{PICK}} \mathrm{n}^{\prime} \mathrm{DROP}=38.04(\mathrm{SD}=2.80)$.

\section{Ratio of Object Transfers per Interaction}

As our interaction techniques allowed participants to change the ratio of object transfers per interaction (e.g., picking up three sticky notes sequentially and then dropping them all on another tablet), we analyzed if this ratio varied across the conditions. Here, we define $l$ as a 1:1 ratio (i.e., picking up one sticky note and dropping it afterward) and 5 as a 5:1 ratio (i.e., picking up five sticky notes and dropping them afterward at once). For TRAY, the ratio could be varied by e.g., dragging multiple sticky notes via multiple fingers out of it. A statistical analysis revealed no statistically significant differences with $\mathrm{M}_{\mathrm{MENU}}=1.64(\mathrm{SD}=0.67), \mathrm{M}_{\mathrm{TRAY}}=2.40(\mathrm{SD}=1.57)$, and $\mathrm{M}_{\text {PICK'n'DROP }}=1.79(\mathrm{SD}=1.14)$.

\section{Time-weighted Tablet Utilization}

We analyzed participants' utilization of the provided tablets using the time-weighted tablet utilization score (TUS, see [35] for further reference). The TUS relates the number of used tablets to their usage time and the overall task completion time. In our case, a TUS of 1.0 would indicate the usage of one tablet for the entire session. Similarly, a TUS of 8.0 would show a $100 \%$ usage of eight tablets. Our analysis revealed a mean TUS of $5.75(\mathrm{SD}=1.07)$ for MENU, $5.01(\mathrm{SD}=1.63)$ for TRAY, and $5.74(\mathrm{SD}=1.45)$ for PICK'n'DROP. Statistical analysis revealed no statistically significant differences. 


\section{Task Load}

We used a NASA TLX questionnaire [10] to investigate participants' task load. A statistical analysis revealed no statistically significant differences for the overall NASA TLX score with $\mathrm{M}_{\mathrm{MENU}}=27.47(\mathrm{SD}=16.44), \mathrm{M}_{\mathrm{TRAY}}=19.79(\mathrm{SD}=12.16)$, and $\mathrm{M}_{\text {PICK'n'DROP }}=25.73(\mathrm{SD}=17.35)$.

We found a statistically significant difference for Physical Demand $\left(\chi^{2}(2)=7.649, p<.05\right)$. A post-hoc analysis showed statistically significant differences for a comparison of TRAY and PICK'n'DROP $(Z=-2.732, p<.016)$ with $\mathrm{M}_{\mathrm{TRAY}}=16.46$ $(\mathrm{SD}=16.58)$, and MPICK'n'DROP $=27.50(\mathrm{SD}=24.89)$.

\section{Subjective Rating}

We asked participants to rank the techniques based on their subjective preferences. For analysis, we gave interaction techniques scores based on their ranked position (e.g., 1 for the technique ranked best, 2 for the one ranked second, and 3 for the least preferred technique). The mean scores are: $M E N U$ : 2.71 (SD=0.55), TRAY: 1.67 (SD=0.70), and PICK'n'DROP: $1.62(\mathrm{SD}=0.71)$. The overall analysis showed a statistical significant difference $\left(\chi^{2}(2)=18.083, p<.05\right)$. A post-hoc test showed a statistically significant difference for pairwise comparisons of TRAY and MENU $(Z=-3.376, p<.016)$ and PICK'n'DROP and MENU $(Z=-3.542, p<.016)$.

PICK'n'DROP was rated best by 12 participants. Participants described it as "like picking up real objects" and that it was "fun", "fast", and its "fluent interaction" was "intuitive". Eleven participants rated TRAY as their favorite interaction technique - they liked the "familiar handling" of drag interactions, described it as "like a game", and found it "interesting to see sticky notes appear on other tablets". 18 ranked MENU to be the least preferred technique. Although described as "you don't have to think, it's like on a PC", participants disliked the additional sub-tasks to invoke the context MENU itself.

RQ1 - Findings: The three interaction techniques partly influenced participants' performance: TRAY and PICK'n'DROP are participants' choice, and both are faster than MENU - with PICK'n'DROP showing a higher physical demand than TRAY.

\section{RQ2 - Work Behavior | Abstract Classification Task}

To investigate work behavior, we focus on our video analysis and resulting types of work behaviors.

\section{Results of Video Analysis}

Table 1 shows an overview of our three video codes in relation to the three interaction techniques. All conditions show an emphasis on Distributing, which can be directly mapped to the task: It was necessary to distribute all sticky notes to solve it. Analyzing this quantitative comparison using Friedman tests revealed no statistically significant differences. Yet, our additional qualitative analysis revealed differences in participants workflows that cannot directly be linked to one of the interaction techniques. We discuss these types of work behaviors from a qualitative perspective in the next subsection.

\begin{tabular}{l||l|l|l} 
& MENU & TRAY & PICK'n'DROP \\
\hline \hline Sorting & $16.96 \%(13.43)$ & $20.33 \%(21.62)$ & $16.38 \%(19.55)$ \\
\hline Distributing & $71.96 \%(15.29)$ & $66.42 \%(20.89)$ & $71.96 \%(22.63)$ \\
\hline Exploring & $3.00 \%(10.24)$ & $4.13 \%(13.08)$ & $3.25 \%(14.12)$
\end{tabular}

Table 1. Mean values of relative duration of activities. Remaining relative duration (about $8 \%$ ) were used to arrange workplaces. Standard deviation (SD) is shown in brackets.

\section{Types of Work Behaviors}

Analyzing the frequencies, sequences, and duration of the three different video codes helped us to get a rich understanding of participants' work behaviors. Although the quantitative analysis did not reveal any differences, we were able to identify three types (T1 - T3) of problem-solving approaches by analyzing all 72 sessions ( 24 participants $\times 3$ conditions) qualitatively: 1) T1: One Tablet, Many Piles, 2), T2: Many Tablets, One Pile Each, and 3) T3: Mixed.

T1: One Tablet, Many Piles - Here, sorting of sticky notes was the main activity. Participants piled up sticky notes with the same letter on the initial tablet. They creatively circumvent the spatial limitations of a single tablet (e.g., it was possible to arrange six sticky notes as a grid without overlaps, but they moved piles at/over the edge of the tablet to overcome spatial restrictions) and moved every "new" sticky note to its corresponding pile. Eventually, participants came up with eight piles, reflecting the eight different letters/classes of sticky notes. Then, they transferred each pile to other tablets.

T2: Many Tablets, One Pile Each - Here, participants skipped sorting and instantly transferred each top-layer sticky note (the sticky note that was shown on top of the pile of all sticky notes) to another tablet. They worked through the initial pile of sticky notes and finished the task when all sticky notes were distributed. Rather than sorting sticky notes on a single tablet, they used the affordances of physical devices as containers.

T3: Mixed - This type of working behavior combines attributes from the other types. Participants either explicitly or implicitly sorted sticky notes: When sorting explicitly, participants piled up sticky notes - similar to $T 1$ - but transferred them to another tablet either when there was no additional space for new piles on the initial tablet or as soon as a pile was complete (e.g., all five "E" sticky notes are piled up). Transferring them to another tablet freed space on the initial tablet, which was then used again to pile up other sticky notes. When sorting implicitly, participants were looking for a specific letter - exploring the sticky notes while spreading them.

In total, $14 \%$ of all sessions were classified as $T 1,32 \%$ as $T 2$, and $54 \%$ as $T 3$. When linking the different types of work behavior to our three different interaction techniques, we found that 16 out of 24 participants acted as a T3 using MENU. For TRAY, there were 10 T3, 9 T2, and 5 T1. Using PICK'n'DROP, there were $12 \mathrm{~T} 3,9 \mathrm{~T} 2$ and $3 \mathrm{Tl}$.

Additionally, our qualitative observations allowed us to gain insights how participants utilized the large device ecology: 11 participants spread all tablets before they started with the actual task in all three conditions, 5 participants always added 


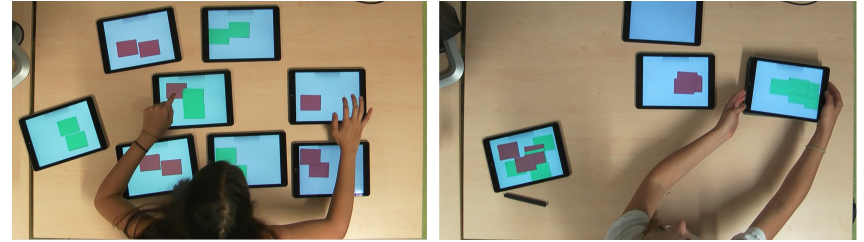

Figure 5. A participant uses both hands (MENU, left) and a participant stacks tablets (right).

tablets incrementally, and 8 participants switched from an incremental workflow in their first condition to spreading all tablets initially in the remaining conditions. Further, participants used both hands to speed up their activity and stacked 'finished' tablets (see Fig. 5).

RQ2 - Findings: From a quantitative perspective, there were no differences between the interaction techniques regarding the amount of time they explored, sorted, and distributed sticky notes. Our qualitative analysis revealed not only problem-solving approaches that participants used to solve the task across all techniques, but also various subtleties of cross-device interaction with a large ecology of devices.

\section{RQ3 - Cross-Device Interrelations | The Murder Mystery}

Here, we differentiate between frequencies of using the interaction techniques, participants' tablet utilization, and participants' rationales for incorporating the interaction techniques into their workflow for the second task. After completion of the murder mystery task, participants were asked five questions about the plot. For analysis of the results, we scored participants' correct answers. On average, participants answered $3.96(\mathrm{SD}=1.17)$ out of 5 questions correctly, indicating that the task was challenging enough, yet not too demanding. The mean task completion time was $911,08 \mathrm{~s}(\mathrm{SD}=469,44)$, and participants worked with $7.38(\mathrm{SD}=0.97)$ tablets on average to solve the task.

\section{Number of Interactions}

We analyzed the number of interactions that were used to solve the task, the times participants used one of the three interaction techniques for it, and how often one of the techniques was chosen by participants. On average, participants transferred sticky notes 48.35 ( $\mathrm{SD}=9.70)$ times to solve the task.

As we did not restrict participants in their usage of the interaction techniques, we analyzed the number of interactions per technique. Also, it was possible to combine the interaction techniques (e.g., picking up a sticky note using PICK'n'DROP and placing it on another tablet using MENU) - thus, we divided this analysis accordingly. On average, participants used MENU 3.29 ( $\mathrm{SD}=9.42$ ) times to cut and 3.63 ( $\mathrm{SD}=9.41)$ times to paste sticky notes. They used TRAY $21.19(\mathrm{SD}=22.59)$ times, and PICK'n'DROP was used 23.88 (SD=23.56) times to pick and $23.46(\mathrm{SD}=23.25)$ times to drop sticky notes. 16 out of 24 participants combined the different interaction techniques and incorporated them into their workflow. Six par-
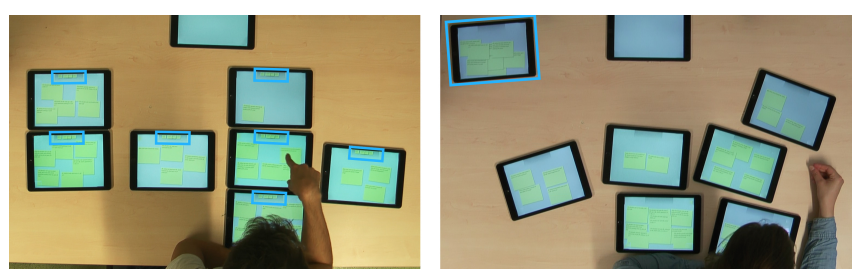

Figure 6. Participants dealing with non-important pieces of information: Repurposing TRAY (left) and a dedicated tablet as "trash bin" (both highlighted in blue).

ticipants used MENU, 20 participants used TRAY, and 17 participants used PICK'n'DROP.

\section{Tablet Utilization}

We analyzed participants utilization of the provided tablets using the time-weighted tablet utilization score (TUS) [35]. Our analysis revealed a mean TUS of $6.50(\mathrm{SD}=0.99)$.

\section{Work Behavior}

We did a qualitative analysis of the video data to see if the codes used for the abstract task and thus the different types of work behaviors also fit to the sensemaking task. Here, we rarely saw sorting activities - participants focused on distributing sticky notes to available tablets and clustering them semantically e.g., by persons or places. This work behavior is similar to the $T 2$ behavior, as participants used the physical affordances of the tablets as containers for different topics. This is further reflected in participants' TUS: Here, the TUS for the sensemaking task (6.50) indicates an early utilization of a large number of tablets and was even higher than in the abstract classification task.

\section{Subjective Rationales for Using the Interaction Techniques}

As a final step, we asked participants about their usage of the three different interaction techniques. Participants had to provide reasons for (not) using each of the interaction techniques.

Participants liked MENU because it is "fun", "easy", and it was the "fastest technique to move sticky notes from one tablet to another". Participants used it to "select sticky notes that are related to persons and collect them on a single tablet." Participants chose MENU instead of PICK'n'DROP in case of "overlapping sticky notes" - indicating spatial limitations of PICK'n'DROP. Participants valued the utilization of both hands, as it was "more natural to use both hands and thus, more fun and faster". Yet, participants criticized the integration into the workflow and did not use MENU because of "too many steps", which made it "inconvenient". Participants "didn't like it" because it "was easier to stick to one technique" and "sticky notes disappeared" when cut - highlighting the importance of the visibility of system status [32].

Participants liked TRAY because it was "handy", they used it to "sort sticky notes on different tablets", and found it "easy to distribute information on different displays." They used it when they were "aware where a sticky note fits", but also to "get needless sticky notes out of view" and called it a "trash bin" (see Figure 6). On the other hand, they did not use TRAY because sticky notes had to be moved "sequentially" (integration into the workflow), they thought they would "have forgotten 
content while being in the TRAY" (visibility of system status) because the task "was too complex" for using TRAY.

Participants liked PICK'n'DROP because they wanted to "cherish information", "the technique fits best to the task", and it "feels natural". They also described it as "fun and you can use both hands", as "a fast way to move information", and it was "useful to move multiple sticky notes at the same time." Interestingly, they mentioned that "it feels like picking up a real object" and that "having this feeling of holding a sticky note in your right hand hinders you to drop it with the left hand, because it's in the other hand!" (utilization of both hands). Participants did not use PICK'n'DROP because using "TRAY was easier" and "more practical" and they chose to use "TRAY, because I wanted to focus on the murder mystery" (integration into the workflow).

RQ3 - Findings: Our analysis showed that participants utilized a large number of tablets to solve the task at hand. The TUS for task 2 was even higher than in task 1. This high score might be based on extensive training during the first task. Participants' choice of interaction techniques for the sensemaking task reflect their subjective preferences for the abstract classification task. We identified quality features for successful cross-device interaction based on participants' rationales for using the techniques: Visibility of status, utilization of both hands, and smooth integration into the workflow.

\section{DISCUSSION}

We discuss our findings based on the topics legacy bias and work behaviors, quality features for successful cross-device interaction, and reflect on our holistic evaluation approach using different types of tasks and both, qualitative insights and quantitative measurements. We additionally provide implications for future research and design.

\section{Legacy Bias and Work Behavior}

Plank et al.'s [35] evaluation of a collaborative multi-tablet system revealed that participants worked with one or two tablets to solve an analytic task. The authors link this finding to a legacy bias, indicating that participants are influenced by their pre-existing knowledge with prior interfaces and technologies. They question if an extensive training in using multiple devices (similar to Morris et al.'s priming technique [28]) can facilitate users in utilizing more devices and thus, reduce the legacy bias (one of the key challenges for future cross-device interaction [4]). Based on our results, we identified two ways that might help to reduce this legacy bias: 1) Guidance and 2) Training. We guided participants in using a large number of tablets with our abstract classification task. Participants had to utilize all available tablets to solve the task. This showed on the one hand the apparent fact that participants do utilize larger numbers of devices if they have to, yet, on the other hand, this can lead to different work behaviors - characterized by utilizing multiple devices at different points in time. We believe that guiding users is a potential way to reduce the legacy bias by learning the benefits of utilizing multiple devices, each with distinct purposes while solving the task at hand.
Our qualitative analysis revealed three types of work behaviors: 1) T1: One Tablet, Many Piles, 2) T2: Many Tablets, One Pile Each, and 3) T3: Mixed. While working with the abstract classification task, participants were aware that they would not be surprised by the contents of sticky notes on the initial pile. It was clear that there are sticky notes with eight different letters that could be explored, sorted, and distributed. This can explain individual differences in work behavior. The different types of work behavior can also be at least loosely linked to the legacy bias and the transaction costs [14] of utilizing multiple tablets into the workflow. Participants had to involve all available devices at some point in time. The $T l$ types were more tentative in utilizing multiple tablets at an earlier stage, compared to the $T 2$ and $T 3$ types that both involved other tablets after a short period of time. This differentiation in three types of work behavior was not present in the sensemaking task: Here, all participants focused on distributing activities (i.e., utilizing a larger number of devices early), e.g., to gain an overview, find suspicious activities, and finally come up with a solution. This behavior is connected to the lack of knowledge of the content of the piled sticky notes but might also be related to the previous training phase of working three times with a large device ecology during the abstract classification task.

\section{Quality Features for Successful Cross-Device Interaction} The importance of the visibility of system status and transparency of interaction was emphasized: Participants complained that "sticky notes disappeared" in MENU and in PICK'n'DROP. Using those techniques, participants had to remember which sticky notes were currently in a non-visual clipboard state - a state that was always visible in TRAY. We favored efficiency [41] over habits as participants were able to transfer multiple sticky notes at the same time - common practice in WIMP interfaces is to only allow one element (e.g., a text fragment) in clipboard state. Participants liked this feature and described that "it fits to the task". Although this additional cognitive effort was not reflected in terms of mental demand measured via a NASA TLX, we believe that the number of elements in clipboard state should be balanced for the task at hand. In our case, participants were perfectly capable of dealing with this feature as it fits with the abstract classification task - participants only had to remember single letters of a limited set. Remembering additional contextual information in the murder mystery or other tasks requires more cognitive capacities and thus should be supported in terms of visibility and transparency either via a visual clipboard (as in TRAY), an additional overview device [3], or by limiting the number of elements in clipboard state.

Participants successfully made use of bi-manual approaches to solve both tasks. They emphasized the "naturalness of using both hands", which was "more fun and faster". Although this is a clear contrast to transferring objects in the real world and also described for PICK'n'DROP ('It's in my other hand!"), some participants valued this possibility, and thus, it shows a reality vs. expressive power tradeoff [17]. We argue that crossdevice interaction techniques should be carefully designed to be performed by both dominant and non-dominant hands. 
Performing interaction techniques requires effort. Participants not only rated PICK'n'DROP with higher physical demand, but they also mentioned that it "was easier to stick to one technique" (MENU), the murder mystery "was too complex" for using TRAY, and they did not use PICK'n'DROP because they "wanted to focus" on the task at hand. Although there was no difference in participants' subjective task load when comparing the interaction techniques, we argue for carefully designing cross-device interaction techniques that interweave with the workflow and to allow focusing on the task at hand.

We observed participants not only creatively repurposing TRAY as a trash bin, but they also used dedicated tablets as trash container - focusing on the essential pieces of information. Additionally, they mentioned that TRAY was particularly helpful when "aware where a sticky note fits". In this user study, all available tablets looked the same - with no differences in color, shape, or size. Although Conductor's color association was not perceived as beneficial [9], we think future research could investigate the influences of different form factors of multi-device environments to see if the combined utilization of e.g., different sized mobile devices leads to the association of different purposes (e.g., a trash bin).

\section{Reflections on the holistic evaluation approach}

Brudy et al. [4] discuss strategies to evaluate cross-device interactions and emphasize the need for more work on evaluation methods to "support human activities in a cross-device ecology or to meaningfully compare cross-device interaction techniques [4]." We tried to tackle the separation of current evaluation strategies by employing different but complementary angles of evaluation that allowed us to study aspects of usability extended by insights into work behaviors.

User studies in HCI research usually involve specific tasks depending on the research focus - ranging from rather artificial workflows to complex sensemaking activities. However, the task itself, besides the apparatus, is typically considered as one of the limitations of a study. We tried to loosen this limitation by including two different task types in our user study: While the abstract classification task allowed us to independently investigate the different interaction techniques and their effect on participants' performance and work behavior, the sensemaking task helped us to explore how the trained techniques are adopted and how a large ecology of devices is utilized. With this holistic evaluation approach, we did not only combine different strategies but also made a first step to compare cross-device interaction techniques meaningfully.

We believe that the methodological combination of different types of tasks in a user study is an effective way to gain different perspectives on a research problem. Future research should further investigate the effects and influences of suitable task combinations allowing complementary insights into participants' usage of cross-device approaches. This might eventually result in components of a universal testbed or evaluation framework that enables to compare techniques or systems and creates a frame of reference for future research.

\section{LIMITATIONS AND FUTURE WORK}

One of the limitations of our study is the focus on tablet-sized devices. Similar to recent research that has shown the influences of device sizes on interactions (e.g., [47, 48]), mobile devices with a smaller size (e.g., mobile phones) or larger tablets could lead to differences in our identified working behaviors smaller devices could favor distributing activities due to spatial limitations; sorting activities could potentially benefit from larger devices. Also, the desk environment limits the generalizability of our results: Participants were free in choosing spatial configurations of devices that suited their needs, yet they were still bound to the size of the desk, the seating arrangement, and the desk itself as a surface to arrange devices. Interesting work behavior could evolve when participants are also free to move around the desk or can arrange devices vertically (as seen on murder boards on TV) including handheld devices and additional pens for annotations, especially for sensemaking activities. Furthermore, allowing participants to work with such a large ecology of devices not only in a controlled lab environment but also in their familiar surroundings (i.e., in the wild) might lead to additional, complementary quality features of cross-device interaction. Although we integrated two task types in our user study that allowed us to see the bigger picture, different tasks types or task duration might lead to different results: The utilization of devices might differ for a) tasks with dedicated or pre-defined devices for various activities (e.g., creative tasks), b) the quantity of information (e.g., visual analytics), and c) longer task duration might extend behaviors (e.g., different purposes for devices).

\section{CONCLUSION}

In this paper, we studied the interplay of interaction techniques, device utilization, and task-specific work behavior in a crossdevice setting. We conducted a user study with different but complementary angles of evaluation with an abstract classification task [23], a sensemaking task [43], and three different interaction techniques. We studied 24 participants working with our multi-tablet application using a large ecology of devices of eight tablets $\left(9.7^{\prime \prime}\right)$ in total. We evaluated participants' performance (RQ1), their work behavior (RQ2), and the interrelations of interaction techniques, device utilization, and task-specific activities (RQ3). We found that different interaction techniques have a surprisingly lower influence than expected, that work behaviors and device utilization heavily depend on the task at hand, and that participants value certain quality features of cross-device interaction. Our holistic evaluation approach with different task types and measurements allowed us to gain different yet complementary insights into otherwise hidden subtleties of cross-device interaction. We hope our work inspires future research to further investigate the nuances of a large ecology of devices, cross-device interaction, and evaluation strategies for them.

\section{ACKNOWLEDGMENTS}

This research was funded by the Deutsche Forschungsgemeinschaft (DFG, German Research Foundation) - ProjectID 251654672 - TRR 161 (Project C01). 


\section{REFERENCES}

[1] Ignacio Avellino, Cédric Fleury, Wendy E. Mackay, and Michel Beaudouin-Lafon. 2017. CamRay: Camera Arrays Support Remote Collaboration on Wall-Sized Displays. In Proceedings of the 2017 CHI Conference on Human Factors in Computing Systems (CHI '17). ACM, New York, NY, USA, 6718-6729. DOI: http://dx.doi.org/10.1145/3025453.3025604

[2] Fearn Bishop, Johannes Zagermann, Ulrike Pfeil, Gemma Sanderson, Harald Reiterer, and Uta Hinrichs. 2019. Construct-A-Vis: Exploring the Free-Form Visualization Processes of Children. IEEE Transactions on Visualization and Computer Graphics (2019), 1-1. DOI: http://dx. doi .org/10.1109/TVCG.2019.2934804

[3] Frederik Brudy, Joshua Kevin Budiman, Steven Houben, and Nicolai Marquardt. 2018. Investigating the Role of an Overview Device in Multi-Device Collaboration. In Proceedings of the 2018 CHI Conference on Human Factors in Computing Systems (CHI '18). ACM, New York, NY, USA, Article 300, 13 pages. DOI: http://dx.doi.org/10.1145/3173574.3173874

[4] Frederik Brudy, Christian Holz, Roman Rädle, Chi-Jui Wu, Steven Houben, Clemens Nylandsted Klokmose, and Nicolai Marquardt. 2019. Cross-Device Taxonomy: Survey, Opportunities and Challenges of Interactions Spanning Across Multiple Devices. In Proceedings of the 2019 CHI Conference on Human Factors in Computing Systems (CHI '19). ACM, New York, NY, USA, Article 562, 28 pages. DOI : http://dx.doi.org/10.1145/3290605.3300792

[5] Nicholas Chen, François Guimbretière, and Abigail Sellen. 2012. Designing a Multi-slate Reading Environment to Support Active Reading Activities. ACM Trans. Comput.-Hum. Interact. 19, 3, Article 18 (Oct. 2012), 35 pages. DOI :

http://dx.doi.org/10.1145/2362364.2362366

[6] Nicholas Chen, François Guimbretière, and Abigail Sellen. 2013. Graduate Student Use of a Multi-slate Reading System. In Proceedings of the SIGCHI Conference on Human Factors in Computing Systems (CHI '13). ACM, New York, NY, USA, 1799-1808. DOI: http://dx.doi.org/10.1145/2470654.2466237

[7] Linda Di Geronimo, Maria Husmann, and Moira C. Norrie. 2016. Surveying Personal Device Ecosystems with Cross-device Applications in Mind. In Proceedings of the 5th ACM International Symposium on Pervasive Displays (PerDis '16). ACM, New York, NY, USA, 220-227. DOI :

http://dx.doi.org/10.1145/2914920.2915028

[8] Georges G. Grinstein, Theresa A. O'Connell, Sharon J. Laskowski, Catherine Plaisant, Jean Scholtz, and Mark A. Whiting. 2006. VAST 2006 Contest - A Tale of Alderwood. 2006 IEEE Symposium On Visual Analytics Science And Technology (2006), 215-216.

[9] Peter Hamilton and Daniel J. Wigdor. 2014. Conductor: Enabling and Understanding Cross-device Interaction.
In Proceedings of the SIGCHI Conference on Human Factors in Computing Systems (CHI '14). ACM, New York, NY, USA, 2773-2782. DOI :

http://dx.doi.org/10.1145/2556288.2557170

[10] Sandra G. Hart and Lowell E Staveland. 1988. Development of NASA-TLX (Task Load Index): Results of empirical and theoretical research. Human mental workload 1, 3 (1988), 139-183.

[11] Leila Homaeian, Nippun Goyal, James R. Wallace, and Stacey D. Scott. 2018. Group vs Individual: Impact of TOUCH and TILT Cross-Device Interactions on Mixed-Focus Collaboration. In Proceedings of the 2018 CHI Conference on Human Factors in Computing Systems (CHI '18). ACM, New York, NY, USA, Article 73, 13 pages. DOI:

http://dx.doi.org/10.1145/3173574.3173647

[12] Tom Horak, Sriram Karthik Badam, Niklas Elmqvist, and Raimund Dachselt. 2018. When David Meets Goliath: Combining Smartwatches with a Large Vertical Display for Visual Data Exploration. In Proceedings of the ACM Conference on Human Factors in Computing Systems. ACM, 13. DOI:

http://dx.doi.org/10.1145/3173574.3173593

[13] Tom Horak, Andreas Mathisen, Clemens N. Klokmose, Raimund Dachselt, and Niklas Elmqvist. 2019. Vistribute: Distributing Interactive Visualizations in Dynamic Multi-Device Setups. In Proceedings of the 2019 CHI Conference on Human Factors in Computing Systems (CHI '19). ACM, New York, NY, USA, Article 616, 13 pages. DOI:

http://dx.doi.org/10.1145/3290605.3300846

[14] Steven Houben, Nicolai Marquardt, Jo Vermeulen, Clemens Klokmose, Johannes Schöning, Harald Reiterer, and Christian Holz. 2017. Opportunities and Challenges for Cross-device Interactions in the Wild. interactions 24, 5 (Aug. 2017), 58-63. DOI: http://dx.doi .org/10.1145/3121348

[15] Kaori Ikematsu and Itiro Siio. 2015. Memory Stones: An Intuitive Information Transfer Technique Between Multi-touch Computers. In Proceedings of the 16th International Workshop on Mobile Computing Systems and Applications (HotMobile '15). ACM, New York, NY, USA, 3-8. DOI : http://dx.doi.org/10.1145/2699343.2699352

[16] British Standards Institution. Test charts for clinical determination of distance visual acuity. 2003. London.

[17] Robert J.K. Jacob, Audrey Girouard, Leanne M. Hirshfield, Michael S. Horn, Orit Shaer, Erin Treacy Solovey, and Jamie Zigelbaum. 2008. Reality-based Interaction: A Framework for post-WIMP Interfaces. In Proceedings of the SIGCHI Conference on Human Factors in Computing Systems (CHI '08). ACM, New York, NY, USA, 201-210. DOI : http://dx.doi.org/10.1145/1357054.1357089 
[18] Mikkel R. Jakobsen and Kasper Hornbæk. 2015. Is Moving Improving?: Some Effects of Locomotion in Wall-Display Interaction. In Proceedings of the 33rd Annual ACM Conference on Human Factors in Computing Systems (CHI '15). ACM, New York, NY, USA, 4169-4178. DOI :

http://dx.doi.org/10.1145/2702123.2702312

[19] Tero Jokela, Jarno Ojala, Guido Grassel, Petri Piippo, and Thomas Olsson. 2015. A Comparison of Methods to Move Visual Objects Between Personal Mobile Devices in Different Contexts of Use. In Proceedings of the 17th International Conference on Human-Computer Interaction with Mobile Devices and Services (MobileHCI'15). ACM, New York, NY, USA, 172-181. DOI : http://dx.doi.org/10.1145/2785830.2785841

[20] Clemens N. Klokmose, James R. Eagan, Siemen Baader, Wendy Mackay, and Michel Beaudouin-Lafon. 2015. Webstrates: Shareable Dynamic Media. In Proceedings of the 28th Annual ACM Symposium on User Interface Software \& Technology (UIST'15). ACM, New York, NY, USA, 280-290. DOI : http://dx.doi.org/10.1145/2807442.2807446

[21] Ricardo Langner, Tom Horak, and Raimund Dachselt. 2018. VisTiles: Coordinating and Combining Co-located Mobile Devices for Visual Data Exploration. IEEE Transactions on Visualization and Computer Graphics 24 (1 2018), 626-636. Issue 1. DOI :

http://dx.doi.org/10.1109/TVCG.2017.2744019

[22] Can Liu, Olivier Chapuis, Michel Beaudouin-Lafon, and Eric Lecolinet. 2016. Shared Interaction on a Wall-Sized Display in a Data Manipulation Task. In Proceedings of the 2016 CHI Conference on Human Factors in Computing Systems (CHI '16). ACM, New York, NY, USA, 2075-2086. DOI :

http://dx.doi.org/10.1145/2858036.2858039

[23] Can Liu, Olivier Chapuis, Michel Beaudouin-Lafon, Eric Lecolinet, and Wendy E. Mackay. 2014. Effects of Display Size and Navigation Type on a Classification Task. In Proceedings of the 32nd Annual ACM Conference on Human Factors in Computing Systems (CHI '14). ACM, New York, NY, USA, 4147-4156. DOI : http://dx. doi .org/10.1145/2556288.2557020

[24] Andrés Lucero, Jussi Holopainen, and Tero Jokela. 2011. Pass-them-around: Collaborative Use of Mobile Phones for Photo Sharing. In Proceedings of the SIGCHI Conference on Human Factors in Computing Systems (CHI '11). ACM, New York, NY, USA, 1787-1796. DOI : http://dx.doi.org/10.1145/1978942.1979201

[25] Nicolai Marquardt, Till Ballendat, Sebastian Boring, Saul Greenberg, and Ken Hinckley. 2012. Gradual Engagement: Facilitating Information Exchange Between Digital Devices As a Function of Proximity. In Proceedings of the 2012 ACM International Conference on Interactive Tabletops and Surfaces (ITS '12). ACM, New York, NY, USA, 31-40. DOI :

http://dx.doi.org/10.1145/2396636.2396642
[26] Nicolai Marquardt, Frederik Brudy, Can Liu, Ben Bengler, and Christian Holz. 2018.

SurfaceConstellations: A Modular Hardware Platform for Ad-Hoc Reconfigurable Cross-Device Workspaces. In Proceedings of the $2018 \mathrm{CHI}$ Conference on Human Factors in Computing Systems (CHI'18). ACM, New York, NY, USA, Article 354, 14 pages. DOI : http://dx.doi.org/10.1145/3173574.3173928

[27] Nicolai Marquardt, Ken Hinckley, and Saul Greenberg. 2012. Cross-device Interaction via Micro-mobility and F-formations. In Proceedings of the 25th Annual ACM Symposium on User Interface Software and Technology (UIST '12). ACM, New York, NY, USA, 13-22. DOI : http://dx.doi.org/10.1145/2380116.2380121

[28] Meredith Ringel Morris, Andreea Danielescu, Steven Drucker, Danyel Fisher, Bongshin Lee, m. c. schraefel, and Jacob O. Wobbrock. 2014. Reducing Legacy Bias in Gesture Elicitation Studies. interactions 21, 3 (May 2014), 40-45. DOI : http://dx. doi .org/10.1145/2591689

[29] Miguel A Nacenta, Carl Gutwin, Dzmitry Aliakseyeu, and Sriram Subramanian. 2009. There and back again: Cross-display object movement in multi-display environments. Human-Computer Interaction 24, 1-2 (2009), 170-229.

[30] Michael Nebeling. 2017. XDBrowser 2.0: Semi-Automatic Generation of Cross-Device Interfaces. In Proceedings of the 2017 CHI Conference on Human Factors in Computing Systems (CHI'17). ACM, New York, NY, USA, 4574-4584. DOI : http://dx.doi.org/10.1145/3025453.3025547

[31] Thomas Neumayr, Hans-Christian Jetter, Mirjam Augstein, Judith Friedl, and Thomas Luger. 2018. Domino: A Descriptive Framework for Hybrid Collaboration and Coupling Styles in Partially Distributed Teams. Proc. ACM Hum.-Comput. Interact. 2, CSCW, Article 128 (Nov. 2018), 24 pages. DOI: http://dx. doi .org/10.1145/3274397

[32] Jakob Nielsen. 1994. Enhancing the Explanatory Power of Usability Heuristics. In Proceedings of the SIGCHI Conference on Human Factors in Computing Systems (CHI '94). ACM, New York, NY, USA, 152-158. DOI : http://dx. doi.org/10.1145/191666.191729

[33] Antti Oulasvirta. 2008. FEATURE: When Users "Do" the Ubicomp. interactions 15, 2 (March 2008), 6-9. DOI : http://dx.doi .org/10.1145/1340961.1340963

[34] Jeni Paay, Dimitrios Raptis, Jesper Kjeldskov, Mikael B. Skov, Eric V. Ruder, and Bjarke M. Lauridsen. 2017. Investigating Cross-Device Interaction Between a Handheld Device and a Large Display. In Proceedings of the 2017 CHI Conference on Human Factors in Computing Systems (CHI '17). ACM, New York, NY, USA, 6608-6619. DOI :

http://dx.doi.org/10.1145/3025453.3025724 
[35] Thomas Plank, Hans-Christian Jetter, Roman Rädle, Clemens N. Klokmose, Thomas Luger, and Harald Reiterer. 2017. Is Two Enough?! Studying Benefits, Barriers, and Biases of Multi-Tablet Use for Collaborative Visualization. In Proceedings of the 2017 CHI Conference on Human Factors in Computing Systems (CHI '17). ACM, New York, NY, USA, 4548-4560. DOI :

http://dx.doi.org/10.1145/3025453.3025537

[36] Roman Rädle, Hans-Christian Jetter, Jonathan Fischer, Inti Gabriel, Clemens N. Klokmose, Harald Reiterer, and Christian Holz. 2018. PolarTrack: Optical Outside-In Device Tracking That Exploits Display Polarization. In Proceedings of the $2018 \mathrm{CHI}$ Conference on Human Factors in Computing Systems (CHI '18). ACM, New York, NY, USA, Article 497, 9 pages. DOI : http://dx.doi.org/10.1145/3173574.3174071

[37] Roman Rädle, Hans-Christian Jetter, Nicolai Marquardt, Harald Reiterer, and Yvonne Rogers. 2014.

HuddleLamp: Spatially-Aware Mobile Displays for Ad-hoc Around-the-Table Collaboration. In Proceedings of the Ninth ACM International Conference on Interactive Tabletops and Surfaces (ITS '14). ACM, New York, NY, USA, 45-54. DOI :

http://dx.doi .org/10.1145/2669485.2669500

[38] Roman Rädle, Hans-Christian Jetter, Mario Schreiner, Zhihao Lu, Harald Reiterer, and Yvonne Rogers. 2015. Spatially-aware or Spatially-agnostic?: Elicitation and Evaluation of User-Defined Cross-Device Interactions. In Proceedings of the 33rd Annual ACM Conference on Human Factors in Computing Systems (CHI '15). ACM, New York, NY, USA, 3913-3922. DOI :

http://dx.doi.org/10.1145/2702123.2702287

[39] Jun Rekimoto. 1997. Pick-and-drop: A Direct Manipulation Technique for Multiple Computer Environments. In Proceedings of the 10th Annual ACM Symposium on User Interface Software and Technology (UIST '97). ACM, New York, NY, USA, 31-39. DOI : http://dx.doi.org/10.1145/263407.263505

[40] Mario Schreiner, Roman Rädle, Hans-Christian Jetter, and Harald Reiterer. 2015. Connichiwa: A Framework for Cross-Device Web Applications. In Proceedings of the 33rd Annual ACM Conference Extended Abstracts on Human Factors in Computing Systems (CHI EA '15). ACM, New York, NY, USA, 2163-2168. DOI : http://dx.doi .org/10.1145/2702613.2732909

[41] Stacey D. Scott, Guillaume Besacier, and Phillip J. McClelland. 2014. Cross-device transfer in a collaborative multi-surface environment without user identification. In 2014 International Conference on
Collaboration Technologies and Systems (CTS). 219-226. DOI :

http://dx.doi.org/10.1109/CTS. 2014.6867568

[42] Mikael B. Skov, Jesper Kjeldskov, Jeni Paay, Heidi P. Jensen, and Marius P. Olsen. 2015. Investigating Cross-Device Interaction Techniques: A Case of Card Playing on Handhelds and Tablets. In Proceedings of the Annual Meeting of the Australian Special Interest Group for Computer Human Interaction (OzCHI '15). ACM, New York, NY, USA, 446-454. DOI: http://dx.doi.org/10.1145/2838739.2838763

[43] Gene Stanford and Barbara Dodds Stanford. 1969. Learning discussion skills through games. New York : Citation Press. 75 pages.

[44] James R. Wallace, Stacey D. Scott, and Carolyn G. MacGregor. 2013. Collaborative Sensemaking on a Digital Tabletop and Personal Tablets: Prioritization, Comparisons, and Tableaux. In Proceedings of the SIGCHI Conference on Human Factors in Computing Systems (CHI '13). ACM, New York, NY, USA, 3345-3354. DOI :

http://dx.doi.org/10.1145/2470654.2466458

[45] Mark Weiser. 1991. The computer for the 21st century. Scientific American 265, 3 (Sept. 1991), 66-75. DOI: http://dx. doi .org/10.1145/329124.329126

[46] Paweł Wozniak, Nitesh Goyal, Przemysław Kucharski, Lars Lischke, Sven Mayer, and Morten Fjeld. 2016. RAMPARTS: Supporting Sensemaking with Spatially-Aware Mobile Interactions. In Proceedings of the 2016 CHI Conference on Human Factors in Computing Systems (CHI '16). ACM, New York, NY, USA, 2447-2460. DOI: http://dx.doi.org/10.1145/2858036.2858491

[47] Johannes Zagermann, Ulrike Pfeil, Daniel Fink, Philipp von Bauer, and Harald Reiterer. 2017. Memory in Motion: The Influence of Gesture- and Touch-Based Input Modalities on Spatial Memory. In Proceedings of the 2017 CHI Conference on Human Factors in Computing Systems (CHI '17). ACM, New York, NY, USA, 1899-1910. DOI :

http://dx.doi.org/10.1145/3025453.3026001

[48] Johannes Zagermann, Ulrike Pfeil, Roman Rädle, Hans-Christian Jetter, Clemens Klokmose, and Harald Reiterer. 2016. When Tablets Meet Tabletops: The Effect of Tabletop Size on Around-the-Table Collaboration with Personal Tablets. In Proceedings of the 2016 CHI Conference on Human Factors in Computing Systems (CHI '16). ACM, New York, NY, USA, 5470-5481. DOI :

http://dx.doi.org/10.1145/2858036.2858224 